\title{
BMJ Open Quality Improving access to City and Hackney adult mental health services
}

David Zekria, Amar Shah, Yunus Malik, Deeksha Mehta, Forid Alom, Amrus Ali, Charles Kennedy-Scott, Andrew Horobin

To cite: Zekria $D$, Shah $A$, Malik Y, et al. Improving access to City and Hackney adult mental health services.BMJ Open Quality 2017;6:e000014. doi:10.1136/ bmjoq-2017-000014

Received 23 January 2017 Revised 19 August 2017 Accepted 4 September 2017

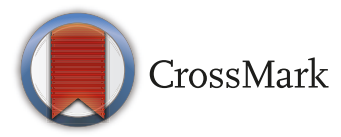

East London NHS Foundation Trust, London, UK

Correspondence to

Dr Amar Shah;

amarshah@nhs.net

\section{ABSTRACT}

City and Hackney Adult Mental Health Referral and Assessment Service (CHAMHRAS) is the single point of entry for all mental health referrals to secondary services, with the exception of perinatal referrals, in the City and Hackney region of London, UK. CHAMHRAS was established in 2013 with the objective of providing a onestop point of referral which screens urgent and non-urgent referrals of adults aged 18-65 to mental health services. This single point of entry simplifies the referral process to secondary mental health services-something service users have requested. It also enables rapid feedback on all referrals taken from general practitioners as well as other sources. The centralised nature of CHAMHRAS has also facilitated the monitoring of waiting times from receipt of referral to first face-to-face assessment across services. It was noted that the waiting time for the majority of patients was exceeding the 28-day target set by local commissioners. Indeed, in December 2014 , only $30 \%$ of patients were being seen within this time frame. The aim of this quality improvement project has been to decrease the average waiting time from referral to first face-to-face assessment, and concomitantly increase the proportion of patients being assessed within the 28-day target period. The team identified potential sources of delay in the process of handling referrals, from receipt and triage, to forwarding to the relevant secondary service, and have tested change ideas such as the implementation of daily meetings to review referrals and the centralisation of appointment bookings to streamline the processes and minimise delays. The average waiting time from referral to first face-to-face assessment decreased by $34 \%$ and the proportion of patients being assessed within 28 days increased accordingly, exceeding $95 \%$ in the case of referrals from general practitioners (GP). We have listed changes that we intend to introduce with the aim of bringing waiting times down further.

\section{PROBLEM}

In December 2014, under 30\% of patients were being assessed within the 28-day target time frame (figure 1), with the average waiting time being 58 days. Throughout 2014 , the average waiting time by month varied between 42 and 67 days (figure 2). The project aim was for $95 \%$ of patients to be given an appointment for face-to-face assessment with a healthcare practitioner within non-specialist services in City and Hackney within 28 days of receipt of their referral.
The benefits of prompt assessment include addressing the needs of the patient in a timely manner and therefore improving the experience of patients and their carers, reducing the risk to patients, improving staff satisfaction, improving GP satisfaction and meeting commissioner requirements.

\section{BACKGROUND}

East London NHS Foundation Trust (ELFT) provides mental health services within the boroughs of the City of London, Hackney, Newham and Tower Hamlets, and from April 2015 also in Luton and Bedfordshire. ELFT also provides community health services in Newham, and specialist services to a much wider area.

City and Hackney Adult Mental Health Referral and Assessment Service (CHAMHRAS) is the single point of entry for mental health services in the City and Hackney region, catering to a population of $245000 .{ }^{1}$ GPs and other agencies can contact CHAMHRAS for advice and to make referrals in order to access mental health services. CHAMHRAS is based within the City and Hackney Centre for Mental Health, and the team is made up of nurses, social workers, psychiatrists, support workers and administrators. The service works closely with other teams such as the home treatment team, recovery teams and psychological services.

CHAMHRAS receives referrals, typically via fax or email. The triage team would aim, within 48 hours, to review the referral for urgency and appropriateness, assess the patient if urgent and then admit or directly hand over to an appropriate specialist service.

A delay may be incurred prior to triage if it is necessary to contact the referrer to gather more information, or to contact the person referred and their family. CHAMHRAS would conduct a weekly meeting to review referrals and 'signpost' them to the appropriate specialist service. Holding such meetings on a weekly basis means that while recently received referrals would be attended 


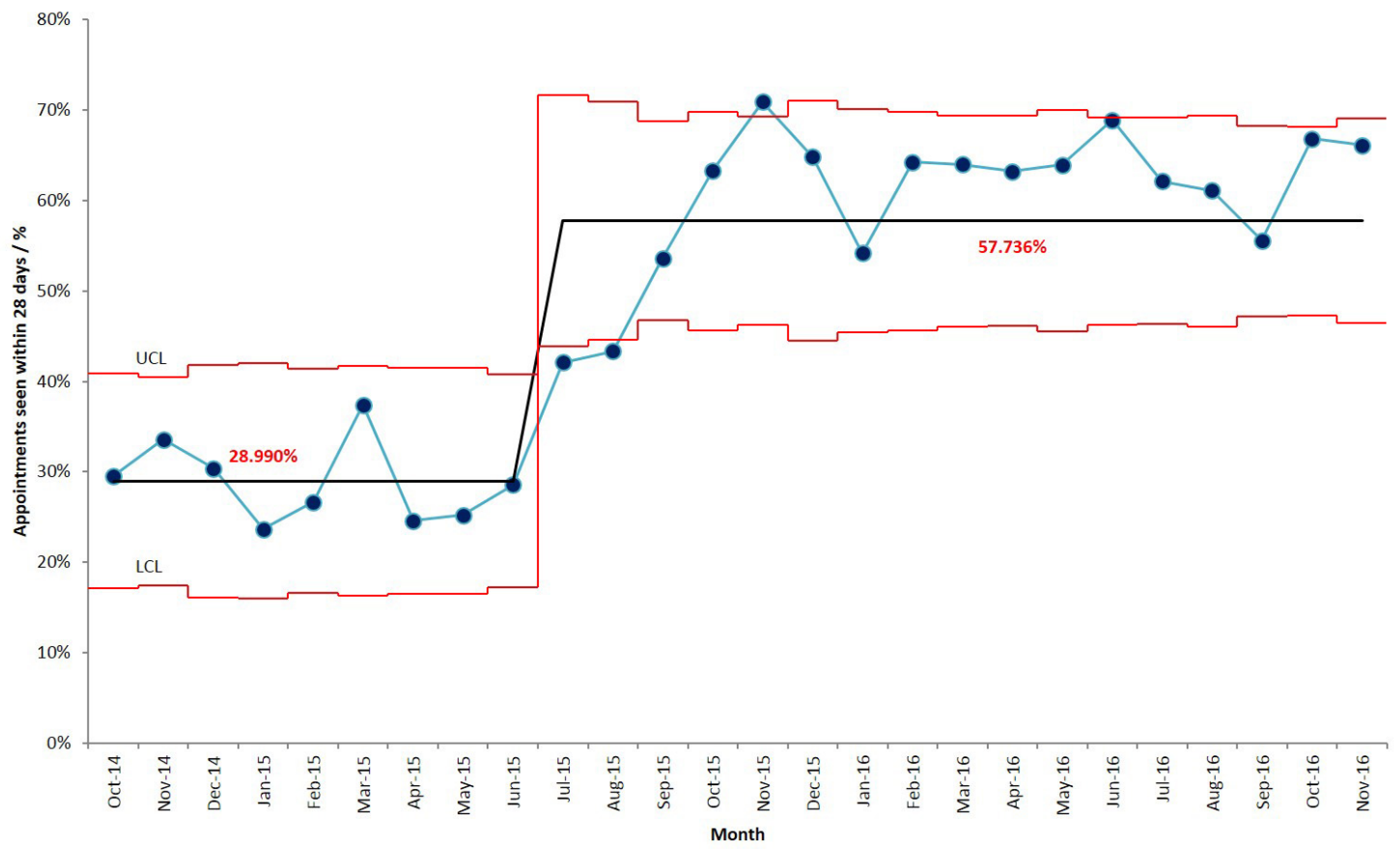

Figure 1 Control chart ( $p$ chart) showing percentage of adult mental health referral appointments seen within 28 days by City and Hackney community mental health teams, as monthly averages, from October 2014 to November 2016. Referrals from all sources are included. UCL and LCL are the upper and lower control limits, respectively. The black line shows the mean.

to quickly, others could potentially be waiting for up to 7 days until the next meeting, thereby contributing to the overall waiting time to assessment. Arranging appointments with the various services, each of which have their own appointment management systems, was also time consuming and inefficient.

In order to reduce the waiting time from referral to appointment, the team addressed each of these potential sources of delay, testing change ideas using the PDSA ('Plan, Do, Study, Act') cycle framework and making adaptations based on the learning.

As far as we are aware, no similar projects related specifically to secondary mental health services have been published, though reviews of strategies affecting waiting times for other specialities have been documented. ${ }^{23}$

\section{BASELINE MEASUREMENT}

Two outcome measures were chosen for this project:

- the average waiting time from referral to first face-toface appointment, including both internal and external referrals and including patients who 'Did Not Attend' their appointments (DNAs) and cancellations;

- the percentage of patients who are assessed face-toface within 28 days of receipt of referral; for the latter measure, the clock was reset if the patients cancelled or did not attend their appointment.

In some instances, if an appointment with the relevant service was not available such that the patient could be assessed within 28 days of receipt of referral, the patient would be fast-tracked to assessment in an 'overspill clinic' which was set up to avert breaching of the waiting time target. The number of patients referred to the overspill clinic was monitored as a process measure in this project. As balancing measures, the following were monitored:

- the percentage of non-attendances at appointment; this was calculated as the number of non-attendances divided by the total number of appointments booked, excluding cancellations;

- the number of referrals to CHAMHRAS, from both internal and external sources.

Included in these measures are all referrals which resulted in assessment by a consultant team, a CHAMHRAS worker or by Enhanced Primary Care (EPC). Referrals which progressed to assessment by specialist services were not considered, as specialist services manage their own appointments and have their own waiting time targets.

Data were sourced from the CHAMHRAS database which tracks all referrals and their outcomes. The team overseeing the project liaised with the local performance leads and shared the data with the clinical team in order to ensure that the data being extracted were accurate. A detailed operational definition was developed for each of the measures, so that the clinical team were entering data in an accurate and consistent way.

\section{DESIGN}

Daily referral meeting

It was decided to hold daily referral meetings to remove the inevitable $1-7$ day wait that arises from weekly referral meetings. 


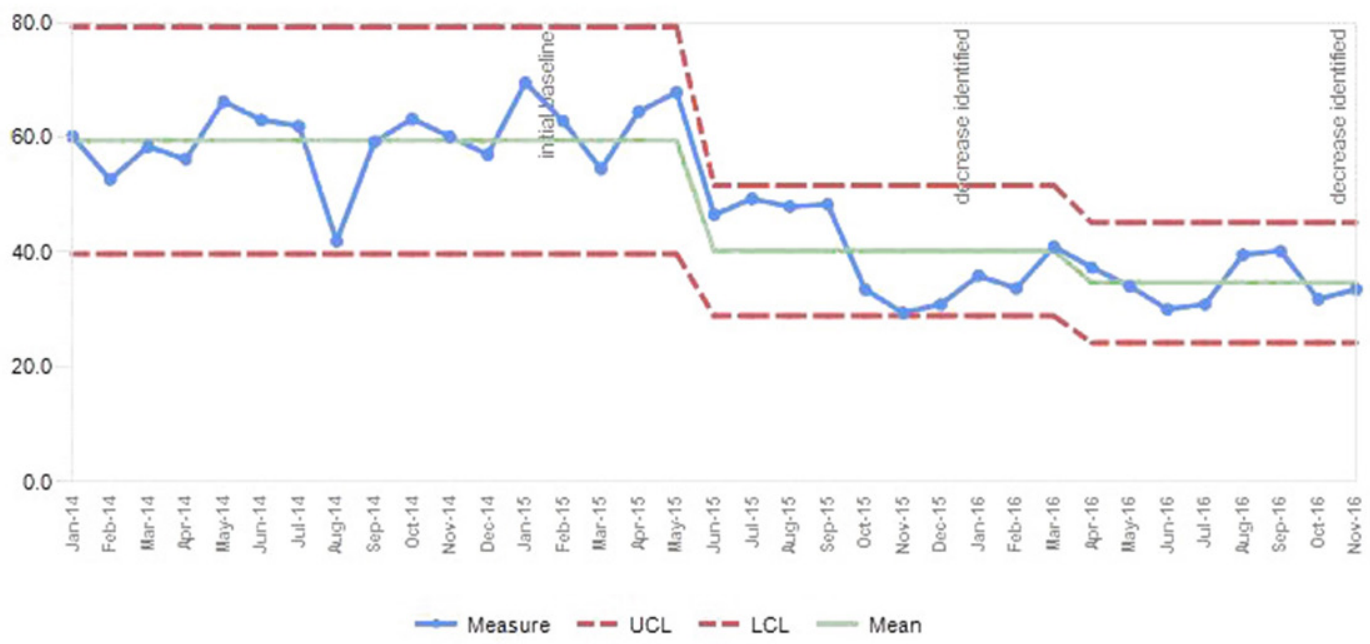

Figure 2 Control chart (i chart) showing average waiting time from referral to first appointment, by month, from January 2014 to November 2016. The measure is the average waiting time in days; UCL and LCL are the upper and lower control limits, respectively; the mean is of the monthly average values, recalculated for periods where the first four monthly values lie below the mean for the preceding period.

\section{Centralised booking of first appointments}

CHAMHRAS coordinates appointment bookings within locality teams' diaries, and for those patients who cannot be booked into slots within 28 days of referral, arranges assessment in an overspill clinic run by CHAMHRAS. Locality teams immediately book new referrals into available slots. (Locality team can test the RiO (electronic patient record system) diary, Outlook Diary or other diaries.)

\section{STRATEGY}

\section{Daily referral meeting}

This step was tested and implemented in a series of three PDSA cycles, starting in August 2015. In the first cycle, daily referral allocation meetings were held at CHAMHRAS to review new referrals received. In the second cycle, the PDSA was rerun with just the North Recovery Team consultants, and process measures were studied and acted upon weekly. The third PDSA cycle was extended to include the South Recovery Team consultants.

\section{Data cleansing}

At the start of the project, it was recognised that some user records had not been closed or had outcomes recorded incorrectly in $\mathrm{RiO}$ and this adversely skewed our performance data. The project team issued guidance on recording practices and supported this with frequent validation exercise to improve data accuracy. Data cleansing was initiated in October 2014.

\section{Implementation of DNA protocol and reminder service}

We identified that different teams had varying tolerance levels for frequent non-attenders which impacted on our capacity to manage referrals. Guidance was issued to encourage teams to discharge users back to GPs after a second DNA or cancellation.

To reduce our DNA rates within the service, we launched text reminders as well as reminder calls 2-7 days prior to appointment to encourage service users to attend appointment or reschedule if required. A frequent DNA case list was circulated and monitored locally by the team as well as overall DNA rates within the service.

This DNA protocol was instituted in February 2016.

\section{Centralised booking of first appointments}

This step was implemented in two PDSA cycles starting in December 2015, in the first cycle with centralised booking of assessment appointments in the North Locality Team's consultants' clinics, and in the second cycle extended to the South Team.

A fundamental principle of the central booking process was that no user was permitted to be booked outside of 28 days, unless all alternatives to find or create capacity were explored. This involved reviewing our communication process within CHAMHRAS to ensure all teams were aware of any potential breaches before they occurred and regularly prompts were set up to improve visibility and compliance.

\section{RESULTS}

We started from an average waiting time baseline of 60.7 days, based on data from 2014. By March 2016, this had reduced to an average of 40.1 days, equivalent to a $34 \%$ reduction in average waiting time (figure 2 ).

Over the same period, the monthly number of referrals to CHAMHRAS grew from a baseline of 176.4 to 385.3 


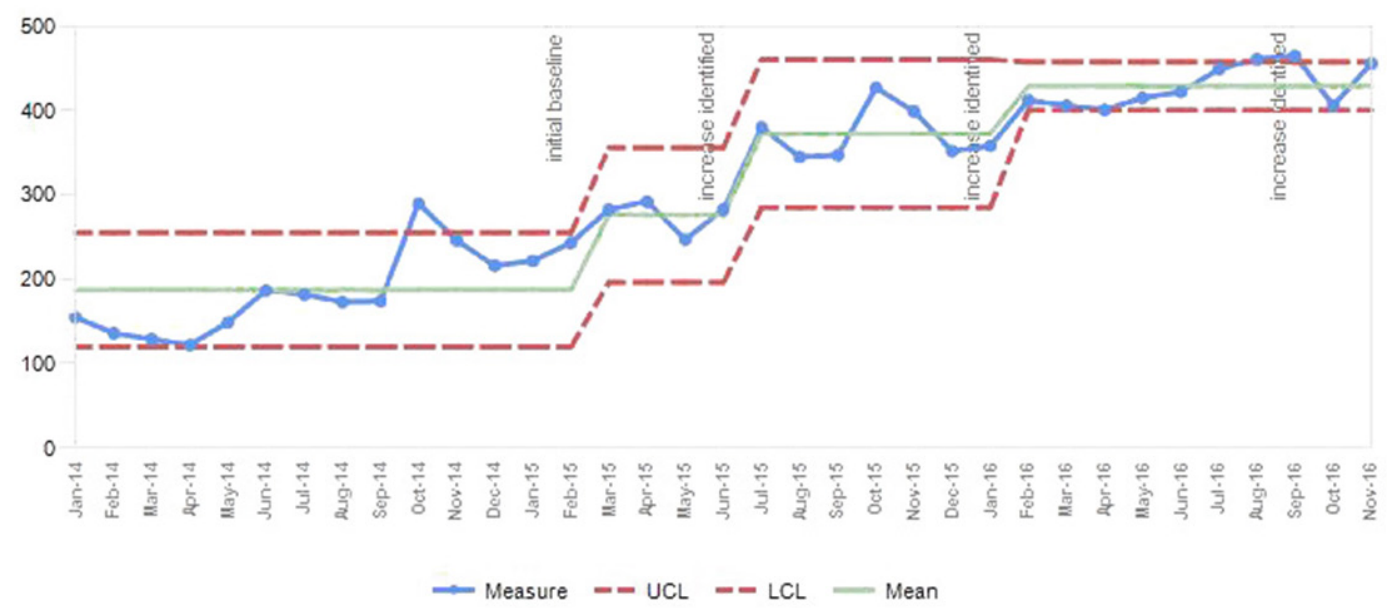

Figure 3 Control chart (i chart) showing number of referrals received by CHAMHRAS (City and Hackney Adult Mental Health Referral and Assessment Service) by month, from January 2014 to November 2016. The measure is the number of referrals; $\mathrm{UCL}$ and LCL are the upper and lower control limits, respectively; the mean is of the monthly values, recalculated for periods where the first four monthly values lie above the mean for the preceding period.

per month (figure 3). The percentage of patients not attending their first face-to-face appointment dropped from a baseline of $51 \%$ to $26 \%$ (figure 4 ).

It was also found that the percentage of non-attendance at first face-to-face appointment positively correlated with the average number of days waited (figure 5). That is to say, the longer the wait from referral to first face-to-face assessment, the greater the likelihood of non-attendance.

There was a negative correlation between the number of referrals accepted and the average number of days waited.
In other words, when the waiting time was reduced, the number of referrals increased (figure 6).

\section{LESSONS AND LIMITATIONS}

The project aim was to streamline the process from receipt of referral to managing assessment appointments, with the key focus of reducing unnecessary delays and thereby minimising waiting times. For this to be achieved,

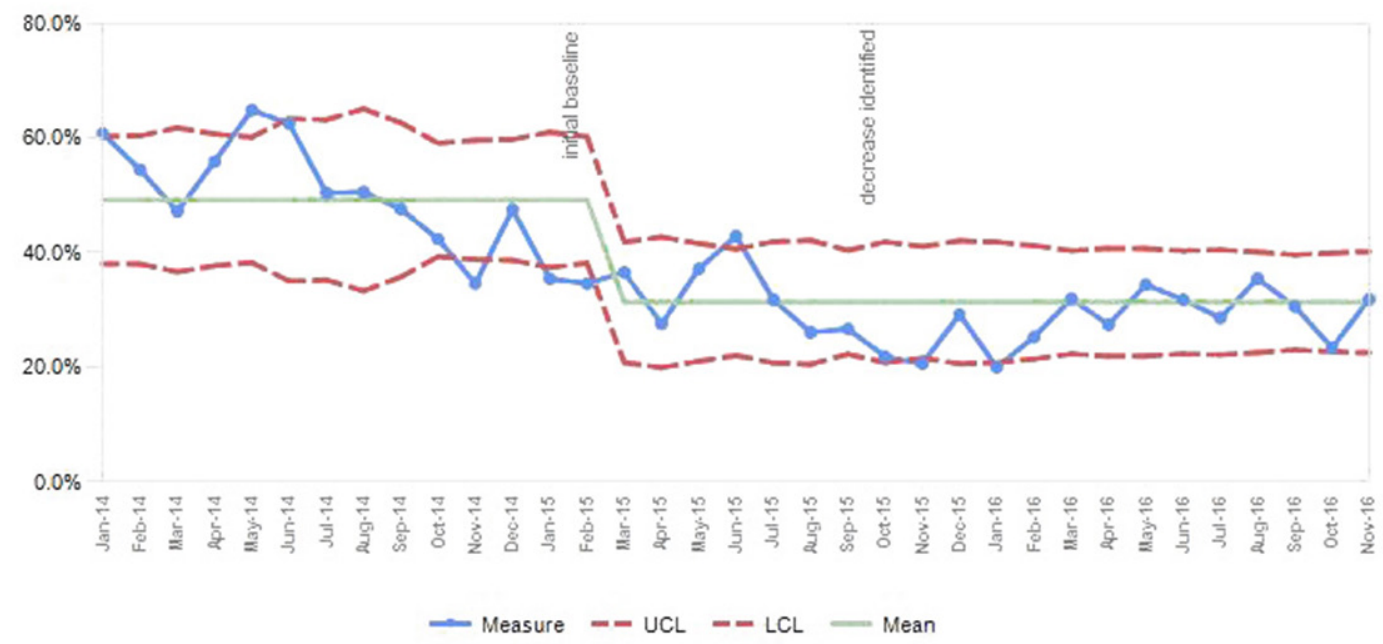

Figure 4 Control chart ( $p$ chart) showing the percentage of first face-to-face appointments not attended, by month, from January 2014 to November 2016. The measure is the percentage of appointments not attended; UCL and LCL are the upper and lower control limits, respectively; the mean is of the monthly values, recalculated for periods where the first four monthly values lie below the mean for the preceding period. 


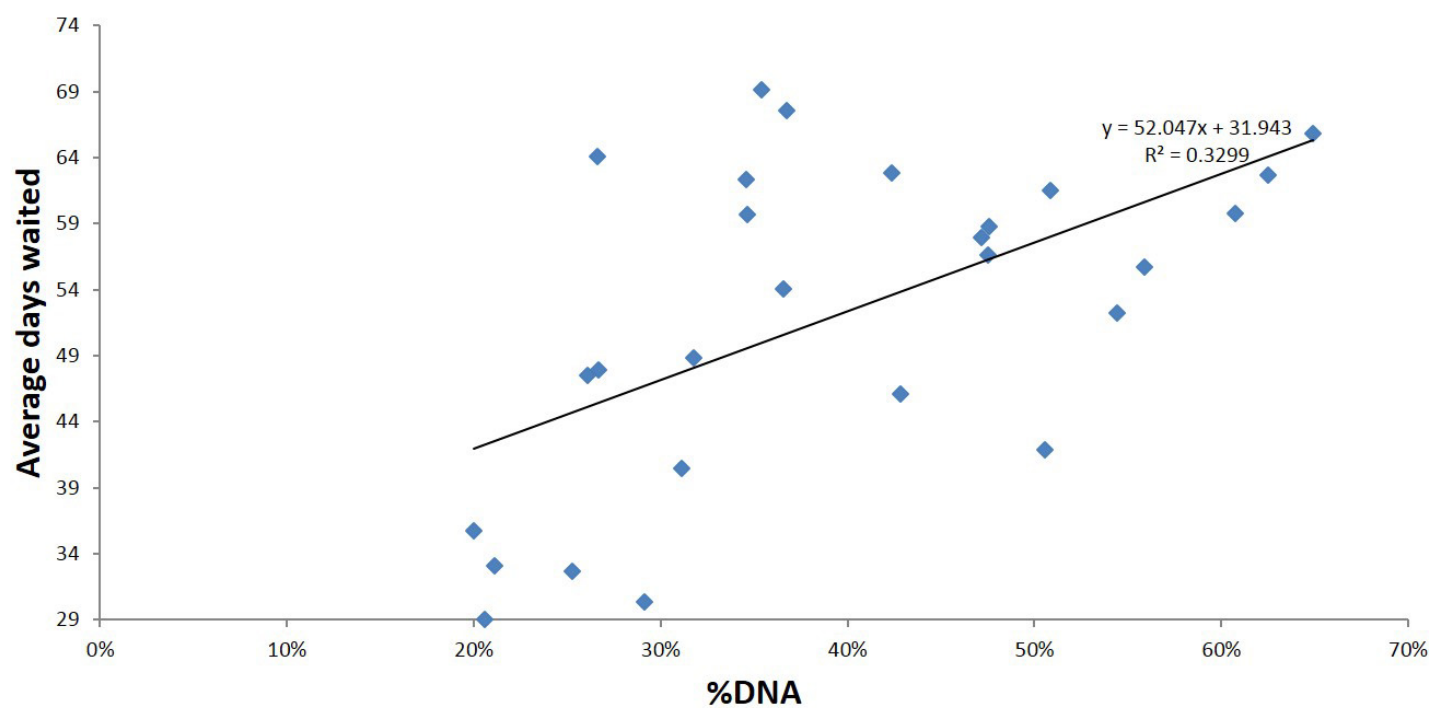

Figure 5 Graph showing the average number of days waited from receipt of referral to first face-to-face appointment versus percentage of appointments not attended ('did not attend', DNA), based on monthly data.

a system needed to be designed that could easily be adopted by the diverse range of participants.

Some challenges were encountered in the implementation of these measures. These included difficulties in engaging teams in subjects like this as no teams have ever looked at their performance in this way before. Data quality was also an issue, though ongoing efforts have been made to clean up the data, to increase the accuracy of what is being reported to staff, patients and commissioners.

Interdisciplinary conflict and securing commitment to test new ways of working has been difficult because of legacy silo working structures and working practices that different teams had embedded. For example, implementing a centralised booking process was complicated by the fact that teams had varying practices of allocating and recording new assessment slots either via $\mathrm{RiO}$, Outlook Diary or paper diaries, and decisions were often determined by clinicians and secretaries on a case-by-case basis. This presented challenges to centralise the entire booking process, but through testing and evaluating alternatives we have managed to take steps towards this and achieving project goals.

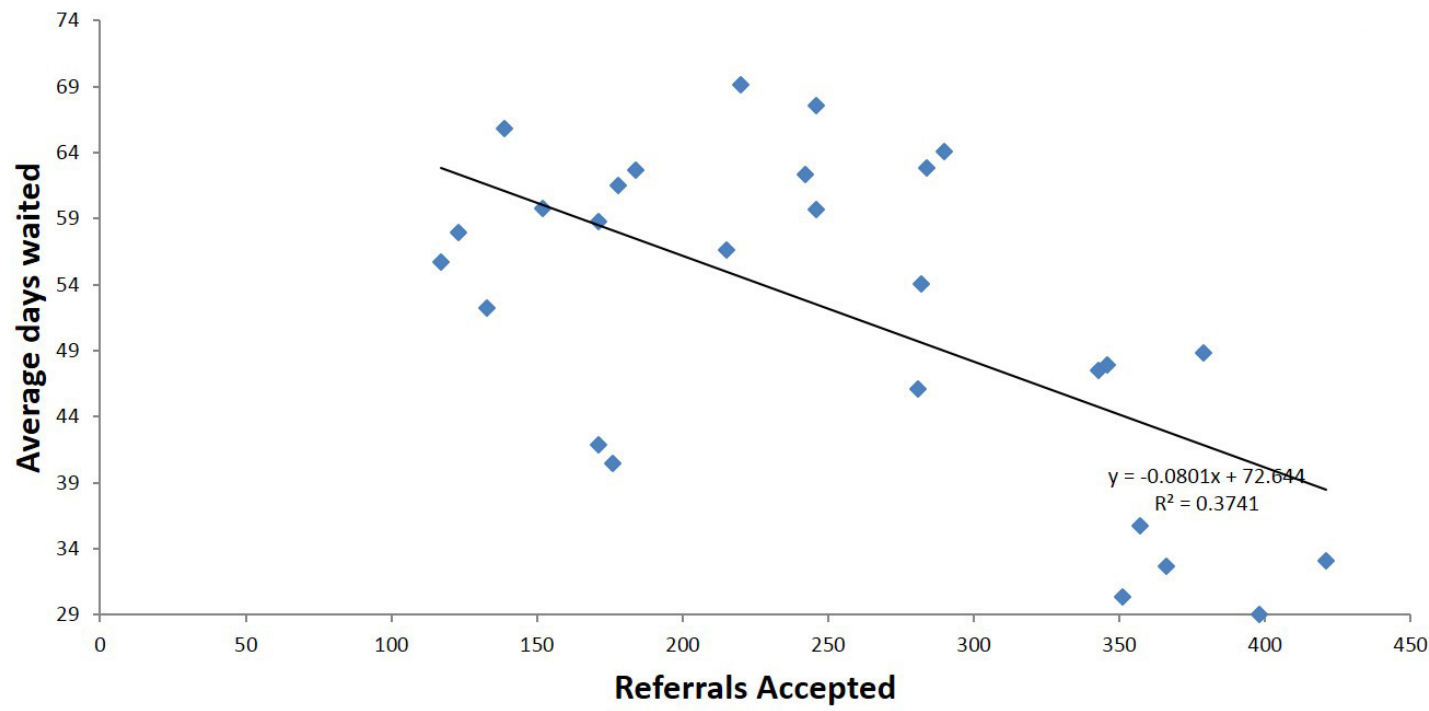

Figure 6 Graph showing the average number of days waited from receipt of referral versus the number of referrals accepted per month, based on monthly data. As referrals have increased, the average waiting time has decreased. 


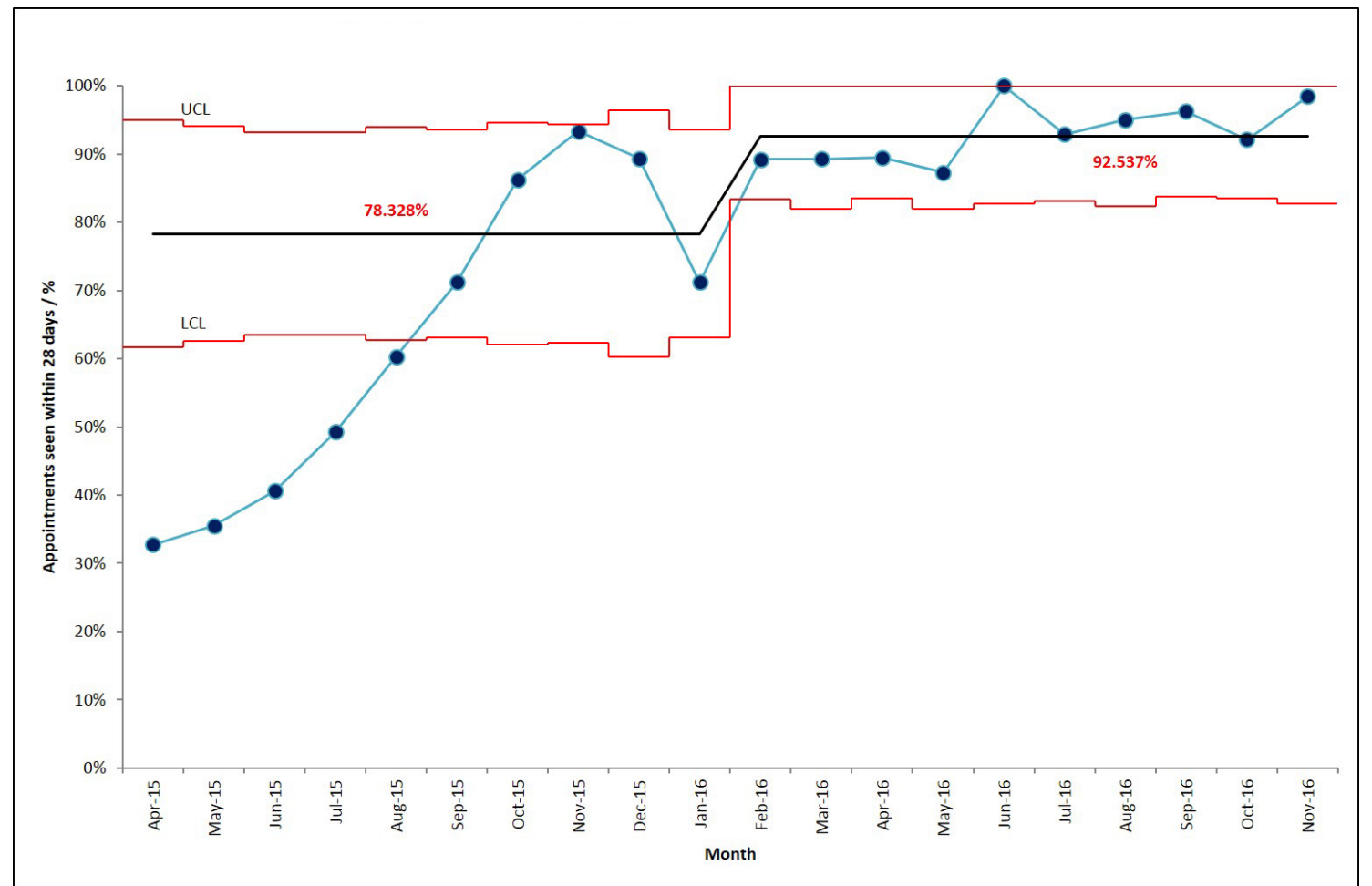

Figure 7 Control chart ( $p$ chart) showing percentage of adult mental health referral appointments seen within 28 days by City and Hackney community mental health teams, as monthly averages, from April 2015 to November 2016. Referrals only from general practitioners are included. UCL and LCL are the upper and lower control limits, respectively. The black line shows the mean.

Multidisciplinary communication was essential to the task, yet coordinating meetings to discuss the project has been a challenge.

We did not understand the whole system until we tried to define it. We are still not entirely clear on the exact capacity in the system. Work is ongoing in this respect. The complexity and size of the project needs to be considered carefully and resources allocated appropriately.

Giving the project high-profile sponsors such as clinical directors helps move the project forward.

\section{CONCLUSION}

The measures implemented have been effective in reducing the average waiting time from referral to initial face-to-face assessment. Likewise, the percentage of patients offered appointments within the 28-day target time frame has increased.

The target of $95 \%$ has been achieved, as of November 2016, for referrals from GPs (figure 7), though still less than $70 \%$ of all referrals are seen within the 28-day target (figure 1).

The measures implemented thus far have been integrated into the normal operation of the service, so we believe they will be sustainable. There are other further changes that we intend to introduce, and we are confident these will reduce average waiting times even more, as well as maximise the percentage of patients assessed within the target time frame. These measures include:
1. developing a standard referral form to ensure relevant information is collected, thus reducing time spent on gathering further information after receipt of the referral, and collecting it in a format compatible with the electronic patient record system, EMIS;

2. some new referrals being assessed by EPC;

3. some new referrals being assessed by non-medical clinicians;

4. review of allocation of GP practices;

5. alternative venues to hold clinics;

6. using a local database to track and manage referrals;

7. using Microsoft Outlook diary and RiO diary for managing appointments;

8. posting overspill clinic appointment letters first class and by CHAMHRAS, thereby reducing administrative time and time in the postal system, giving patients more advanced notice to prepare and thereby reducing the proportion of DNAs;

9. text message reminders for the overspill clinic, with the aim or reducing DNAs;

10. telephone referral system, allowing referrals to be dealt with and 'signposted' more swiftly;

11. refreshing referral criteria;

12. revisiting previous change ideas and ensuring they are implemented and the system can still support them; 
13. working with GP practices to test motivation of users to engage with referrals to secondary care.

Clearly, there is still much scope for CHAMHRAS to optimise the referral process. Even so, this project has been very useful in reducing referral to assessment waiting times.

The team overseeing this project is one of a number of teams at ELFT working on improving access to services, so it constitutes just part of a larger learning system that enables innovation to be shared across teams. We have shared the learning internally through posters, presentations and newsletters to promote the spread of successful change ideas to other contexts. Many of the change ideas tested in this project have been taken up by other teams working on similar challenges in their own services.

Competing interests None declared.
Provenance and peer review Not commissioned; externally peer reviewed.

Open Access This is an Open Access article distributed in accordance with the Creative Commons Attribution Non Commercial (CC BY-NC 4.0) license, which permits others to distribute, remix, adapt, build upon this work non-commercially, and license their derivative works on different terms, provided the original work is properly cited and the use is non-commercial. See: http://creativecommons.org/ licenses/by-nc/4.0/

(C) Published by the BMJ Publishing Group Limited. For permission to use (where not already granted under a licence) please go to http://www.bmj.com/company/ products-services/rights-and-licensing/

\section{REFERENCES}

1. East London NHS foundation trust annual report and accounts ,20152016.

2. Naiker U, FitzGerald G, Dulhunty JM, et al. Time to wait: a systematic review of strategies that affect out-patient waiting times. Aust Health Rev 2017.

3. Ballini L, Negro A, Maltoni S, et al. Interventions to reduce waiting times for elective procedures. Cochrane Database Syst Rev 2015:CD005610. 